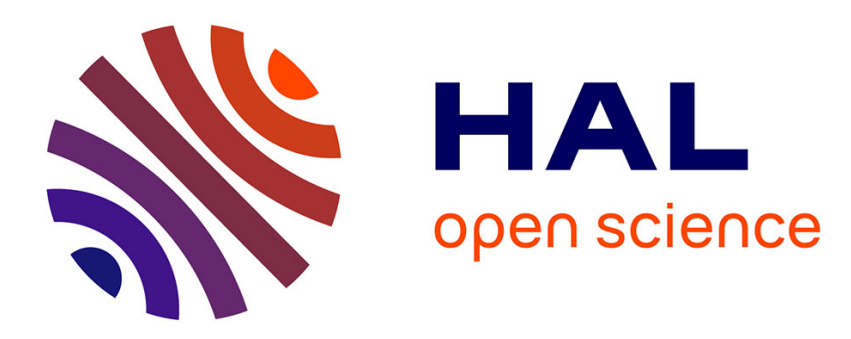

\title{
Probing the nanohydrodynamics at liquid-solid interfaces using thermal motion
}

L. Joly, C. Ybert, L. Bocquet

\section{To cite this version:}

L. Joly, C. Ybert, L. Bocquet. Probing the nanohydrodynamics at liquid-solid interfaces using thermal motion. Physical Review Letters, 2006, 96 (4), pp.046101. 10.1103/PhysRevLett.96.046101 . hal00437085

\section{HAL Id: hal-00437085 \\ https://hal.science/hal-00437085}

Submitted on 9 Dec 2017

HAL is a multi-disciplinary open access archive for the deposit and dissemination of scientific research documents, whether they are published or not. The documents may come from teaching and research institutions in France or abroad, or from public or private research centers.
L'archive ouverte pluridisciplinaire HAL, est destinée au dépôt et à la diffusion de documents scientifiques de niveau recherche, publiés ou non, émanant des établissements d'enseignement et de recherche français ou étrangers, des laboratoires publics ou privés. 


\title{
Probing the Nanohydrodynamics at Liquid-Solid Interfaces Using Thermal Motion
}

\author{
L. Joly, C. Ybert, ${ }^{*}$ and L. Bocquet \\ Laboratoire de Physique de la Matière Condensée et Nanostructures, UMR 5586 Université Claude Bernard Lyon 1 et CNRS, \\ 69622 Villeurbanne Cedex, France
}

(Received 30 June 2005; published 2 February 2006)

\begin{abstract}
We report on a new method to characterize nanohydrodynamic properties at the liquid-solid interface relying solely on the measurement of the thermal motion of confined colloids. This equilibrium measurement of surface properties - equivalent in spirit to the passive microrheology technique used for bulk properties - is able to achieve nanometric resolution on the slip length measurement. Exploring the "zero shear rate" limit, it rules out shear rate threshold to slip effects and extends the range over which slip lengths are shown to be flow independent. Avoiding the nucleation of gas pockets (nanobubbles) through external forcing, it validates the theoretical picture for intrinsic liquid-solid interfaces, reporting nanometric slip lengths $(b=18 \pm 5 \mathrm{~nm})$ only in nonwetting situations, opening the route to quantitative study on more complex surfaces with combined effects of nonwettability and roughness.
\end{abstract}

PACS numbers: 68.08.-p, 47.15.G-, 68.15.+e

During recent years the pursuit of scale reduction inherent to nanotechnologies has been extended to the fluidic domain and liquid flow manipulation, with the important development of micro- and nanofluidics [1]. However, reducing the scale of any system leads invariably to an enhancement of the influence of surface properties with respect to the bulk ones: given the scale reduction, most phenomena take place at the boundaries, and a fundamental understanding of how surface properties might affect the overall flow properties has become crucial to design and optimize operational devices.

Classically, one accounts for the influence of these interfaces through effective boundary conditions (BC) in the description of macroscopic hydrodynamics, the most common of those BC being the no-slip assumption (cf. [3] for an exhaustive review of the literature). However, the possible deviation from this classical hypothesis, resulting in liquid slippage at the solid surface, has recently become a central issue, with immediate perspectives in the microand nanofluidics domains [3] or in the electrokinetic context [2]. Slippage is usually accounted for by a partial slip $\mathrm{BC}$ for the tangential velocity $v_{t}, b \partial_{z} v_{t}=v_{t}$ (with $z$ perpendicular to the planar surface). This generalized BC introduces an extrapolation length $b$, usually denoted as the slip length [4].

The achievement of large (micrometric and above) slip lengths that would directly benefit microdevices, is now mostly sought on composite interfaces [5,6] (so called superhydrophobic substrates), which are able to trap a large amount of gas and therefore replace the actual solid-liquid interface. However, a quantitative understanding and control of such gas-promoted slippage requires prior knowledge of the intrinsic (molecular) slip length on the remaining solid-liquid interface patches [5]. Such a precise knowledge is presently not achieved. From the theoretical and analytical point of view [4,7], the situation is very clear, with no slip occurring on wetting surfaces and with intrinsic slip lengths on nonwetting surfaces not ex- ceeding tens of nanometers and independent of shear rate (over all experimentally accessible values). While this picture is supported by part of the experimental data $[8,9]$, some other data (cf. [3]) completely escape from the theoretical framework, with qualitative (shear rate threshold to slippage; shear rate dependent slip length) and quantitative (micrometric versus nanometric slip length) discrepancies. Up to now, very few proposals are available to explain observed deviation from theoretical expectations, namely, the difficulty in data analysis $[8,10]$ and the presence of trapped gas pockets (often referred to as "nanobubbles" [11-13]) promoted by the measuring flow, preventing access of the intrinsic solid-liquid properties $[5,11,14]$. Clearly, in order to rationalize the experimental picture, new experimental data are necessary. Those data, however, should be gathered in such a way as to avoid previous pitfalls (like measurement-induced gas pockets) and should bring new information on present discrepancies, among which is the shear rate threshold or dependency.

In this Letter, we demonstrate such a new experimental route whereby the nanohydrodynamics of liquids close to surfaces is explored in the absence of any external forcing. Rather than measuring the interfacial dissipation or the forced surface flow, we take advantage of the information already included in response to thermal fluctuations [15] to extract the interfacial dynamics. This technique, which is analogous - for surfaces - to the passive microrheology technique for bulk characterization [16], proves to be extremely sensitive as it allows us to reach an unprecedented resolution for an optical technique, namely, a few nanometers on the slip length measurement. This first "zero shear rate" measurement rules out the existence of shear rate threshold and considerably extends the range over which slip length is shown to be independent of shear rate. Avoiding the possibility of a forced-flow nucleated gas pocket, it completely validates the emerging picture of nanometric slip length $(b=18 \mathrm{~nm})$ for the intrinsic 
properties of nonwetting solid-liquid interfaces [4,8,9] and allows us to address the case of more complex situations combining chemical modification and surface structuration.

We first describe the general principle of our approach. The diffusion dynamics of colloidal tracers is measured in a confined geometry between two solid surfaces of interest, using a homebuilt fluorescence correlation spectroscopy (FCS) device (Fig. 1). Tracer dynamics is affected by confinement, and this dependence reflects the hydrodynamic boundary conditions that apply on both solid substrates [17-19]. Results for different natures of the solid substrates (in particular, varying wettability and surface roughness) lead to measurable differences in the diffusion coefficient, allowing us to deduce the corresponding surface slippage.

Let us now enter into the details of the experimental setup. Colloidal tracers [polystyrene (molecular probes) or silica (Kisker) fluorescent beads with typical diameters $2 a \sim 200 \mathrm{~nm}$ and concentration $\left.c=1 \mathrm{bead} / \mu \mathrm{m}^{3}\right]$ in aqueous solution $\left(10^{-5} \mathrm{M} \mathrm{NaOH} ; 8 \times 10^{-3} M \mathrm{KCl}\right)$ are confined between two solid silica surfaces made from a BK7 spherical lens in contact with a Pyrex plane (Fig. 1). The thermal diffusion dynamics of the colloids is measured in this confined geometry with a FCS device (Fig. 1). With this technique, bead fluorescence is excited with an argon laser focused with a long working distance microscope objective (Leica $40 \times$, numerical aperture of 0.8 ). Fluorescence is then collected through the same objective and sent to a detector (APD, Perkin-Elmer) connected to a correlator via a dichroic mirror and a bandpass filter. While in bulk measurements a confocal pinhole is inserted in the detection pathway to get a spatially defined measurement volume $v$, here the axial limits are set in practice by the two confining walls so that $v=\left(\pi w^{2}\right) e$, with $w$ the beam waist radius and $e$ the wall to wall distance. With a bead concentration such that the mean number of tracers in the detection volume is low (typically around 1 here) fluctuations of the collected intensity $I(t)$ arise due to the motion of beads entering or leaving the measurement volume. The characteristic time scale for such fluctuations corresponds

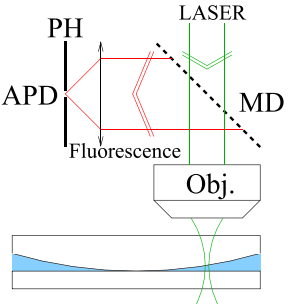

a)

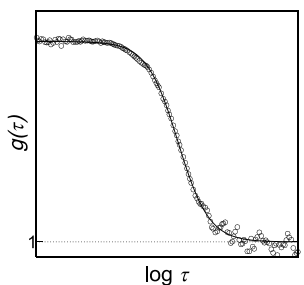

b)
FIG. 1 (color online). (a) Scheme of the experimental cell with FCS detection. Fluorescent colloids suspended in water are confined between a plane and a spherical lens. FCS: (MD) Dichroic mirror, (Obj.) microscope objective, (PH) pinhole, (APD) avalanche photodiode. (b) Typical autocorrelation curve together with theoretical fit according to Eq. (1). to the residence time $\tau_{D}=w^{2} / D$ of a bead within volume $v$, where $D$ is the bead self-diffusion coefficient. More quantitatively, considering a Gaussian radial intensity distribution for the illuminating laser beam, the fluorescence intensity autocorrelation function reads

$$
g(\tau)=\frac{\langle I(t) I(t+\tau)\rangle}{\langle I(t)\rangle^{2}}=1+\frac{1}{n} \frac{1}{\left(1+4 \tau / \tau_{D}\right)},
$$

from which the experimental average number of beads $n$ and their residence time $\tau_{D}$ can be extracted, as shown in Fig. 1. As the bead diffusion coefficient $D$ depends on the location $z$ within the gap, we eventually obtain an averaged value over all the accessible positions within the thickness $e[17,18]$. To ensure good statistics, values for each confinement $e / 2 a$ were accumulated from over 100000 events (associated with one bead passage) split between many different $T=120 \mathrm{~s}$ autocorrelation runs and from at least 5 different experimental cells. Runs belonging to different cells may slightly differ in absolute transit time and average number of beads due to small day to day variations in temperature, concentration, or focus location. We accordingly normalized data from each cell by a reference point $\left(\tau_{0}, n_{0}\right)$ for (almost) unconfined beads, located at $e / 2 a=$ 16.8 , for which excellent statistics was already achieved in the time frame of a single cell experiment. Experimental values from individual runs are normalized by the cell reference point and then averaged for each confinement. Error bars are obtained from the rms of the individual run distribution to which is added the error bar associated with the normalizing reference point.

For any location of the sphere-plane geometry where we measured $n$ and $\tau_{D}$, the wall to wall distance $e$ was simultaneously measured using the interference pattern generated by the two confining surfaces (Newton's rings). Because of the chosen bead size $(2 a \sim 200 \mathrm{~nm})$, data point collection was restricted to the dark fringes (setting a gap increment $\Delta e=183 \mathrm{~nm}$ ), therefore optimizing the signal to noise ratio. Owing to the large radii of curvature of the lenses used (from 250 to $500 \mathrm{~mm}$ ), $e$ is known (and constant over the measurement spot size $2 w \sim 1 \mu \mathrm{m}$ ) to better than $0.4 \%$.

The first element we focused on is the evolution of the mean bead number with the confinement defined as $e / 2 a$. This evolution is shown in Fig. 2 (inset) where we recover a linear behavior for the averaged number of beads in the volume $v$ as expected: $n=\langle c\rangle\left(\pi w^{2}\right) e$. This measurement provides an important check that the beam waist $w$ and the average bead concentration $\langle c\rangle$ are constant over the different measurements' locations. No depletion or adsorption due to bead-surface interaction is therefore detectable in our system, other than a layer of excluded volume close to the surfaces [20]. The thickness of this layer is measured from the extrapolation of the mean beads number down to zero, obtained at $e / 2 a=1.2$ in the inset of Fig. 2. This layer is associated with the additional "excluded volume" resulting from electrostatic repulsions between the wall 


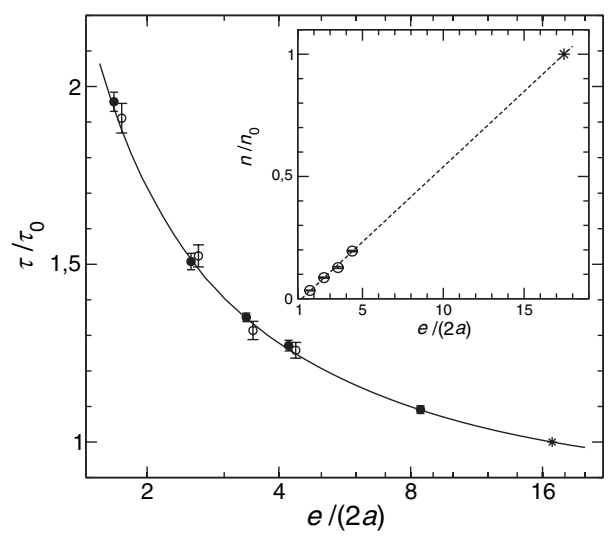

FIG. 2. Evolution of the normalized diffusion time with the confinement $e / 2 a$ between hydrophilic walls for various colloidal tracers: (O) Silica beads $(2 a=218 \mathrm{~nm})$; (O) Polystyrene beads $(2 a=210 \mathrm{~nm})$; (solid line) Theoretical prediction using Femlab and assuming no slip at both walls. Inset: Evolution of the bead number with the confinement. $(\bigcirc)$ Polystyrene beads $(2 a=210 \mathrm{~nm})$; (dotted line) linear regression.

and the beads. Under the present conditions, our experimental measurements using the extrapolation procedure quoted above leads to $d_{\text {excl }} \simeq 20 \mathrm{~nm}$ for bare silica walls (with both polystyrene and silica beads), and a slightly thinner one for silica beads close to silanized walls, $d_{\mathrm{excl}} \simeq$ $13 \mathrm{~nm}$. This lower value of $d_{\text {excl }}$ in the latter case is a signature of the expected decrease of the surface charge on silanized surfaces. The values obtained for $d_{\text {excl }}$ are moreover in good agreement with the estimate obtained from the balance between thermal energy and electrostatic wallbead repulsion energy (calculated using the experimental Debye length and typical zeta potentials for silica).

We now come to the measurement of the residence time - or reciprocally to the diffusion coefficient - of the beads as a function of the confinement $e / 2 a$, for different surface properties. As a starting point, we first considered the case of two hydrophilic confining walls, for which we expect the usual no-slip BC to hold $[4,8,9,21]$. In such a wetting situation, the bead mobility should be strongly reduced by the walls' proximity as was already verified experimentally by a few groups [22,23]. Figure 2 summarizes our results for the measured diffusion times together with theoretical predictions assuming the no-slip boundary condition on both walls. For low confinements $e / 2 a \gg 1$, a very sound, approximate solution for the mobility can be constructed on the basis of the Faxen approximate solution for one wall [18], by adding the independent contribution of each wall to obtain the theoretical bead mobility. However, to avoid theoretical approximations, we conducted numerical resolution of the Stokes equation for a (nonslipping) sphere moving parallel to the two confining solid walls [each characterized by a (possibly different) slip length $b$ ]. A finite element method was implemented using Femlab $\odot$ (see [18] for details). This theoretical prediction for the mobility was then averaged over accessible $z$ (from $a+d_{\text {excl }}$ to $e-a-d_{\text {excl }}$ ) for comparison with experiments $[17,18]$. We, however, point out that changing $d_{\text {excl }}$ in this averaging procedure leads to very weak modifications of the averaged diffusion coefficient, which are quantitatively below our experimental error bars. The changes in diffusion we are measuring are, indeed, mostly a signature of the hydrodynamic long-ranged influence of the confining walls, which does affect the whole fluid thickness [18]. As is evidenced in Fig. 2, the agreement between experiments and theory with no-slip BC on the walls is excellent up to the strongest confinements. Moreover, this agreement is obtained for two types of beads with very different chemistry, showing again that our system is free from specific surface-bead interactions, beside the measured electrostatic repulsion leading to $d_{\text {excl }}$.

We now turn to nonwetting surfaces, for which very different partial slip behaviors have been reported [3]. For that purpose the silica plane was covalently coated with hydrocarbon chains using octadecyltrichlorosilan (OTS). The resulting residence time of silica beads is plotted for different confinement against the previous results for wetting plane (plain silica). When beads are confined enough $(e / 2 a<5)$, residence times are measured to be systematically shorter close to hydrophobic surfaces than to hydrophilic ones. This effect is more easily captured when normalizing this evolution by the theoretical behavior in the absence of slip: $D_{\text {expt }}(e / 2 a) / D_{\text {no-slip }}(e / 2 a)$. A failure of the no-slip boundary condition should result in a departure of this ratio from 1 . This is, indeed, what is observed in the inset of Fig. 3 where the systematic trend described above is best evidenced. Moreover, it is possible to capture accurately this departure by introducing a finite slip length $b$ in the theoretical calculations $[18,19]$. The fitted behavior agrees remarkably well with the experimental data providing a slip length on smooth OTS coated silica planes of $b=18 \pm 5 \mathrm{~nm}$. This "zero shear rate" value is identical to the one obtained in Ref. [8] on the very same surfaces, for shear rates up to $5 \times 10^{3} \mathrm{~s}^{-1}$ and in agreement with recent predictions based on noninvasive neutron reflectivity measurements [24]. In the present situation, no external forcing is applied, thus avoiding nucleation or interaction of flow with gas pockets. We therefore probe the intrinsic (native) liquid-solid hydrodynamic properties. Our experimental results definitely rule out the possibility of a shear rate dependency to slippage in these conditions; be it a threshold or a slip length variation.

We eventually show that slippage on hydrophobic surfaces is strongly modified by the presence of moderate roughness of the solid substrates. Coupling roughness to (non)wettability, the effective slippage on such composite surfaces will strongly depend on their ability to trap gas pockets [5]. As a first step in this direction, we have tested here the combination of hydrophobicity (OTS coated silica) and moderate roughness $[3 \mathrm{~nm}$ rms roughness due to isolated $40 \mathrm{~nm}$ high spikes, obtained by soaking silica planes $30 \mathrm{~min}$ in $\left(1 \mathrm{vol} \mathrm{H}_{2} \mathrm{O}_{2}, 2\right.$ vol $\left.\mathrm{H}_{2} \mathrm{SO}_{4}\right)$ [25] prior to silanization]. When compared to previous smooth hydro- 


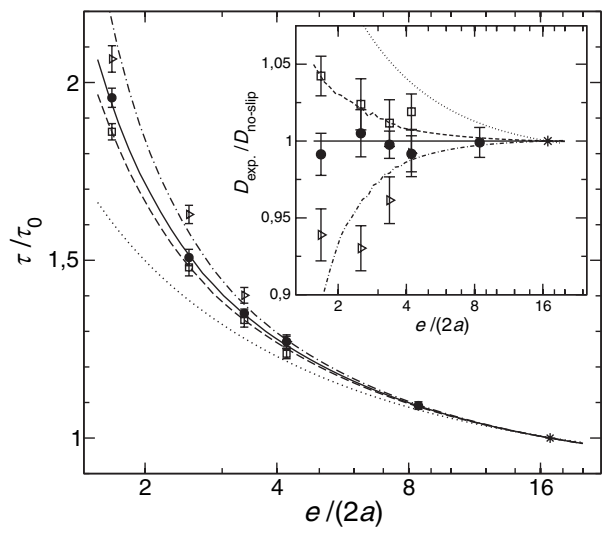

FIG. 3. Evolution of the normalized diffusion time of silica beads $(2 a=218 \mathrm{~nm})$ with confinement between a hydrophilic silica lens and a planar surface with varying surface property: (O) smooth ( $<1 \mathrm{~nm}$ peak-to-peak roughness), hydrophilic surface; $(\triangleright)$ smooth hydrophobic wall (OTS coated silica); ( $\square$ ) rough (40 nm peak-to-peak roughness) hydrophobic wall (OTS coated silica). Theoretical predictions are obtained using Femlab, assuming no slip on the lens, and different $\mathrm{BC}$ on the plane: (solid line) no slip; (dashed line) partial slip $b=18 \mathrm{~nm}$; (dash-dotted line) no slip at a distance $z_{s}=40 \mathrm{~nm}$ from the optically determined surface location (see text for details). As a guide, the dotted line shows the theoretical prediction for a $b=$ $100 \mathrm{~nm}$ slip length, illustrating the method sensitivity.

phobic surfaces $(<1 \mathrm{~nm}$ peak to peak), Fig. 3 shows that bead mobility is strongly reduced close to the rough hydrophobic surfaces. This corresponds to the expected roughness influence in the absence of trapped bubbles between the spikes $[5,10,26]$. In such a case, a rough interface (even slippery) amounts to a smooth nonslipping one, located at a position $z_{s}$ between roughness spikes. In our measurement, the surface optical location (as determined from $e$ ) is expected to be close to the bottom of the roughness (due to the small average contribution of the spiky structure), and we, indeed, found that experimental data are well accounted for by a no-slip surface located at $z_{s}=40 \pm$ $20 \mathrm{~nm}$ from the optical surface location (and therefore close to the top of the roughness) [27].

In this Letter, we have shown that the thermal motion of confined colloidal tracers allows one to characterize the nanohydrodynamics of simple liquids close to surfaces, at "zero shear rate," and with an excellent (nanometric) accuracy. This complementary approach provides information able to clarify experimental controversies concerning the intrinsic liquid-solid slippage: this slippage is shown to occur only on nonwetting surfaces, with a nanometric $b=$ $18 \pm 5 \mathrm{~nm}$ slip length that is shear rate independent from zero shear rate and up to $5 \times 10^{3} \mathrm{~s}^{-1}$ [8]. Exploring a more complex situation with combined roughness and hydrophobicity, it shows that moderate (nanometric) roughness only leads to increased surface friction (in line with a very recent work [10]) and that the pursuit of giant (micrometric) slip lengths necessitates superhydrophobic surfaces with designed roughness to trap a massive amount of gas $[5,6]$. We believe our technique, where no forced flow can interact with gas pockets, will prove valuable for the understanding and tuning of such composite interfaces.

We thank E. Charlaix, C. Cottin-Bizonne, and J.-L. Barrat for stimulating discussions. We acknowledge support from French Ministry for Research (ACI Nanoscience) and Région Rhone-Alpes (projet Emergence).

*Electronic address: cybert@1pmcn.univ-lyon1.fr

[1] G. Whitesides and A. Stroock, Phys. Today 54, No. 1, 42 (2001).

[2] L. Joly, C. Ybert, E. Trizac, and L. Bocquet, Phys. Rev. Lett. 93, 257805 (2004).

[3] E. Lauga, M. Brenner, and H. Stone, in Handbook of Experimental Fluid Dynamics (Springer, New York, to be published); cond-mat/0501557.

[4] J.-L. Barrat and L. Bocquet, Phys. Rev. Lett. 82, 4671 (1999).

[5] C. Cottin-Bizonne, C. Barentin, E. Charlaix, L. Bocquet, and J.-L. Barrat, Eur. Phys. J. E 15, 427 (2004).

[6] J. Ou, B. Perot, and J. P. Rothstein, Phys. Fluids 16, 4635 (2004).

[7] P. Thompson and M. O. Robbins, Phys. Rev. A 41, 6830 (1990).

[8] C. Cottin-Bizonne, B. Cross, A. Steinberger, and E. Charlaix, Phys. Rev. Lett. 94, 056102 (2005).

[9] O. I. Vinogradova and G. E. Yakubov, Langmuir 19, 1227 (2003).

[10] O. I. Vinogradova and G. E. Yakubov (to be published).

[11] P.-G. de Gennes, Langmuir 18, 3413 (2002).

[12] O. I. Vinogradova, N. Bunkin, N. Churaev, O. Kiseleva, A. Lobeyev, and B. Ninham, J. Colloid Interface Sci. 173, 443 (1995).

[13] J. W. G. Tyrrell and P. Attard, Phys. Rev. Lett. 87, 176104 (2001).

[14] E. Lauga and H. A. Stone, J. Fluid Mech. 489, 55 (2003).

[15] A. Einstein, Ann. Phys. 17, 549 (1905).

[16] T. G. Mason and D. A. Weitz, Phys. Rev. Lett. 74, 1250 (1995).

[17] Y. Almeras, L. Bocquet, and J.-L. Barrat, J. Phys. IV (France) 10, 27 (2000).

[18] A. Saugey, L. Joly, C. Ybert, J.-L. Barrat, and L. Bocquet, J. Phys. Condens. Matter 17, S4075 (2005).

[19] E. Lauga and T. Squires, Phys. Fluids 17, 103102 (2005).

[20] Adsorption was found only when polystyrene beads were used together with a hydrophobic surface: this couple was accordingly avoided in our measurements.

[21] D. Y. C. Chan and R. G. Horn, J. Chem. Phys. 83, 5311 (1985).

[22] L. P. Faucheux and A. J. Libchaber, Phys. Rev. E 49, 5158 (1994).

[23] B. Lin, J. Yu, and S. A. Rice, Phys. Rev. E 62, 3909 (2000).

[24] D. A. Doshi, E. B. Watkins, J. N. Israelachvili, and J. Majewski, Proc. Natl. Acad. Sci. U.S.A. 102, 9458 (2005).

[25] L. D. Eske and D. W. Galipeau, Colloids Surf. A 154, 33 (1999).

[26] S. Richardson, J. Fluid Mech. 59, 707 (1973).

[27] Roughness inhomogeneity over the scale of the probed region is responsible for the increased uncertainty. 